\title{
Contributions to the magnetospheric parallel electric field
}

C.R. Stark, A.P. Cran-McGreehin and A.N. Wright

Accepted for publication in Journal of Geophysical Research: Space Physics. Copyright 2011 American Geophysical Union. Further reproduction or electronic distribution is not permitted

Stark, C.R., et al. 2011. Contributions to the magnetospheric parallel electric field. Journal of Geophysical Research: Space Physics. 116(A7). doi: 10.1029/2010JA016270 


\title{
Contributions to the magnetospheric parallel electric z field
}

\author{
C. R. Stark, A. P. Cran-McGreehin and A. N. Wright
}

3 School of Mathematics and Statistics, University of St Andrews, St Andrews,

4 Scotland, UK.

C. R. Stark, Solar and Magnetospheric Group, School of Mathematics and Statistics, University of St Andrews, St Andrews, KY16 9SS, Scotland, UK. (craig@mcs.st-and.ac.uk) 
5 Abstract. Upward field-aligned currents and their associated parallel elec-

6 tric fields couple the ionosphere to the magnetosphere. It is desirable to know

7 how such a potential drop is distributed along the flux tube, what controls

8 its variation and how it is balanced by the plasma. By considering the mo-

9 tion of the ionospheric and magnetospheric electrons and ions, under the in-

10 fluence of electrostatic and magnetic mirror forces, a quasi-steady state, quasi-

${ }_{11}$ neutral electric field distribution along the magnetic flux tube can be obtained.

${ }_{12}$ A feature of the potential profiles is the occurrence of a potential jump, that

${ }_{13}$ splits the profile into three distinct regions: below the jump; within the jump

${ }_{14}$ and above the jump. Within a kinetic framework, we analyse how the plasma

15 velocity distributions evolve along the flux tube, taking into account iono-

${ }_{16}$ spheric, magnetospheric, mirroring and precipitating electron populations.

${ }_{17}$ By calculating the moments of the governing Vlasov equation we ascertain

${ }_{18}$ what balances the parallel electric field $\left(E_{\|}\right)$and how it is maintained, es-

${ }_{19}$ tablishing a dynamical equilibrium. Our calculations show that (1) Earth-

${ }_{20}$ ward of the jump $E_{\|} \approx-\left(p_{\perp} / e n B\right) \nabla_{\|} B$ associated with the ionospheric

${ }_{21}$ electrons, except for at the base of the $\mathrm{F}$ region where $p_{\|}$contributions be-

${ }_{22}$ come more significant; (2) Within the jump magnetosphere electrons dom-

23 inate and $E_{\|} \approx-(1 / e n) \nabla_{\|} p_{\|} ;(3)$ Above the jump mirroring magneto-

${ }_{24}$ spheric electrons make a principal contribution of $E_{\|} \approx-(1 / e n) \nabla_{\|} p_{\|}$,

${ }_{25}$ with a secondary contribution of $-\left(p_{\perp}-p_{\|}\right) \nabla_{\|} B /(n e)$ becoming compa-

${ }_{26}$ rable beyond $\approx 3 \mathrm{R}_{E}$. Additionally, we found that although the precip-

${ }_{27}$ itating electrons carry the field-aligned current it is the mirroring popula- 
${ }_{28}$ tion that determines where $E_{\|}$is concentrated, and hence where precipitat${ }_{29}$ ing electrons are accelerated. 


\section{Introduction}

30 Parallel electric fields and their associated field-aligned currents are key in coupling the

${ }_{31}$ hot, tenuous magnetosphere to the cool, dense ionosphere. They are responsible for the

32 observed aurora and play a significant role in the global circuitry network surrounding

3з the earth. It remains one of the outstanding problems in magnetospheric physics to fully

${ }_{34}$ understand the origin and structure of parallel electric fields. Observations have suggested

35 that elongated, U-shaped potential structures straddling the magnetic field are responsible

${ }_{36}$ for the parallel fields. It is believed that the required parallel potential drop $(\sim 1 \mathrm{kV})$ is

${ }_{37}$ distributed over a large length scale $\left(\sim 10^{3} \mathrm{~km}\right)$ however, observations of large-amplitude

38 electric fields $(\sim 25$ to $300 \mathrm{mV} / \mathrm{m})$ suggest that in some instances a significant fraction

39 of the total potential drop occurs over a much smaller length scale $(\sim 10 \mathrm{~km})$ close to

40 the earth $(\sim 1000 \mathrm{~km})$ [Mozer and Hull, 2001; Hull et al., 2003a, b; Chaston et al., 2007;

${ }_{41} \quad$ Ergun et al., 2000, 2001, 2002].

${ }_{42}$ A significant challenge is understanding how the electric field varies as a function of ${ }_{43}$ position along the magnetic field and what controls that variation. Initial modelling of ${ }_{44}$ magnetospheric fields by Alfvén and Fälthammer [1963] considered a low density, kinetic

45 plasma in a simple magnetic mirror field and found that the parallel electric field vanishes

${ }_{46}$ if the electrons and ions have the same pitch angle, otherwise charge separation results

${ }_{47}$ and an equilibrium electric field is required to ensure the plasma is quasi-neutral along the

48 magnetic flux tube $[$ Persson, 1963, 1966]. Following similar treatments many have built

49 upon these seminal works incorporating additional physics. Alfvén and Fälthammer [1963]

50 only considered magnetospheric, delta source distributions; Knight [1973] relaxed this sim- 
${ }_{51}$ plification and included Maxwellian source distributions at both the magnetosphere and

${ }_{52}$ the ionosphere. This approach yielded a current-voltage relationship, but did not produce

${ }_{53}$ a potential profile, nor did it consider quasi-neutrality. This deficiency was corrected in

${ }_{54}$ the models adopted by Whipple [1977] and Chiu and Schulz [1978]. Stern [1981] found

${ }_{55}$ that double layers, where the potential varies significantly over a discrete length scale,

${ }_{56}$ were a necessary and unavoidable feature of the solutions. Miller and Khazanov [1993]

${ }_{57}$ produced potential profiles where the source distributions could be manipulated to have

${ }_{58}$ a prescribed degree of anisotropy. Others, such as Vedin and Rönnmark [2004], have used

${ }_{59}$ fluid and fluid-kinetic hybrid models to yield potential distributions and current-voltage

60 relations.

${ }_{61} \quad$ Recently Boström [2003, 2004] studied the distribution of current-driven electrostatic

${ }_{62}$ potentials along auroral flux tubes analytically, taking into account quasi-neutrality, the

${ }_{63}$ kinetic orbital motion of the plasma under the influence of electric and magnetic mirror

${ }_{64}$ forces. Paying careful attention to particle accessibility, Boström calculated a series of

${ }_{65}$ potential profiles that included potential jumps (double layers), where a significant fraction

${ }_{66}$ of the total potential drop along the flux tube occurs over a small length scale comparable

${ }_{67}$ to the Debye length. The altitude of the potential jump is sensitive to the relative density

${ }_{68}$ and temperature of the source ionospheric and magnetospheric populations. The purpose

${ }_{69}$ of this paper is to extend the work of Boström $[2003,2004]$ to investigate how the quasi-

70 neutral electric potential variation is balanced and maintained by the plasma, specifically

${ }_{71}$ the electrons, along the flux tube. We derive the moments of the gyrotropic Vlasov

72 equation in order to ascertain what are the significant contributions to the parallel electric

${ }_{73}$ field. We also resolve the ambipolar nature of the ionospheric plasma. 


\section{Upward field-aligned current model}


96 along the flux tube (or equivalently a prescribed potential drop, $\phi_{M}$ ); (2) some plasma processes continuously replenish plasma at the ionospheric and magnetospheric ends of the

${ }_{98}$ flux tube, with a Maxwell-Boltzmann distribution function; (3) a dynamical equilibrium

99 prevails, where there are no temporal variations; (4) the particles carrying the current 
${ }_{119}$ is constant along a particle trajectory). Hence, given a source distribution at $\zeta_{s}$ and ${ }_{120}$ knowledge of how the trajectories behave under the influence of external forces, then ${ }_{121}$ one can obtain the distribution function at an arbitrary point in space $\zeta$. For a plasma ${ }_{122}$ under the influence of electrostatic and magnetic mirror forces, the particle trajectories ${ }_{123}$ are described by the following equations of motion

$$
\begin{aligned}
W_{\| s}+W_{\perp s} \pm U_{s} & =W_{\|}(\zeta)+W_{\perp}(\zeta) \pm U(\zeta) \\
\zeta_{s} W_{\perp s} & =\zeta W_{\perp}(\zeta)
\end{aligned}
$$

${ }_{124}$ where the plus (minus) sign refers to ions (electrons), for ionospheric species add a tilde.

${ }_{125}$ The first equation is the conservation of particle energy and the second represents con126 servation of the first adiabatic invariant $\mu=\frac{1}{2} m v_{\perp}^{2} / B$. Using equations (1) and (2) we ${ }_{127}$ can determine the populated regions of phase space at an arbitrary point $\zeta$. Particles ${ }_{128}$ originating from the source at $\zeta_{s}$, will occupy the region at $\zeta$ defined by

$W_{\|} \geq\left(\zeta / \zeta_{s}-1\right) W_{\perp} \mp\left(U-U_{s}\right)$

$W_{\|} \geq 0$

$W_{\|} \geq\left(\zeta / \zeta_{c}-1\right) W_{\perp} \mp\left(U-U_{c}\right)$ 
${ }_{136}$ (for the full set of phase space diagrams, including ions, see Boström [2004]). Although 
159 of a potential jump (double layer). The maps of $\rho$ apply within the potential jump where

${ }_{160} \zeta$ is approximately constant and the charge density is only a function of $U$. The location

${ }_{161}$ of the potential jump is found by considering Poisson's equation within the double layer.

${ }_{162}$ Following Boström [2004] and others [Langmuir, 1929; Stern, 1981; Block, 1972] multi-

${ }_{163}$ plying Poisson's equation by $\mathrm{d} U / \mathrm{d} \zeta$ and integrating once yields the necessary criteria for

${ }_{164}$ the position $\left(\zeta_{j}\right)$ of the jump,

$$
\int_{U_{j 1}}^{U_{j 2}} \rho\left(\zeta_{j}, U\right) \mathrm{d} U=\frac{k^{2}}{2}\left[\left(\frac{\mathrm{d} U}{\mathrm{~d} \zeta}\right)_{j 1}^{2}-\left(\frac{\mathrm{d} U}{\mathrm{~d} \zeta}\right)_{j 2}^{2}\right] \approx 0
$$

${ }_{165}$ where $k=\lambda_{D} \mathrm{~d} \zeta / d z$ (approximately constant within the double layer); $\lambda_{D}$ is the Debye 166 length; and $z$ is the dimensional coordinate along the flux tube. If the scale of the ${ }_{167}$ background magnetic field variation is $\sim R_{E}$, then $k \approx \lambda_{D} / R_{E} \ll 1$. As described ${ }_{168}$ by Stern [1981] and Boström [2004], if $E_{\|}$adjacent to the jump is much less than that 169 inside, the integral inside equation (5) is zero to leading order.

Note that Boström [2004] assumes no potential drop between the jump location and the ionospheric end (i.e. $U_{j 1}=0$ ). Our calculation relaxes this simplifying assumption, and resolves the ambipolar structure of the ionospheric plasma.

Given the potential variation $U(\zeta)$, the magnetospheric and ionospheric source distribution functions, and the electron trajectories in phase space, we can calculate the moments of the governing Vlasov equation and ascertain how the parallel electric field is maintained, establishing a dynamical equilibrium. An alternative way of expressing this is that we identify the principal routes through which the electric field modifies the electron motion such that a net charge density is established which satisfies Poisson's equation, or quasi-neutrality, as appropriate. For example, the main effect of the electric field could 
be to accelerate the current carrying electrons, or to redistribute the mirroring electrons, etc.

\section{Parallel electric field}

In this section we derive the moments of the Vlasov equation. Initially, we will suppress the non-dimensional notation to aid a physical appreciation.

\subsection{Derivation of electron fluid equations}

Assuming the plasma to be collisionless and the guiding centre approximation valid, the electrons are described by a gyrotropic distribution function $f=f\left(l, v_{\|}, v_{\perp}, t\right)$ which is a function of the distance along the flux tube, $l$; the parallel guiding centre velocity, $v_{\|}$; the perpendicular speed of the electrons, $v_{\perp}$; and time, $t$. Under the influence of a parallel electric field and the magnetic mirror force, the electron dynamics are described by $m \mathrm{~d} v_{\|} / \mathrm{d} t=-e E_{\|}-\mu \partial B / \partial l$, where $\mu$ is the first adiabatic invariant. In the steady state $f=f\left(l, v_{\|}, v_{\perp}\right)$ and the gyrotropic Vlasov equation becomes

$$
v_{\|} \frac{\partial f}{\partial \ell}-\left(\frac{e E_{\|}}{m}+\frac{v_{\perp}^{2}}{2 B} \frac{\partial B}{\partial \ell}\right) \frac{\partial f}{\partial v_{\|}}+\frac{v_{\|} v_{\perp}}{2 B} \frac{\partial B}{\partial \ell} \frac{\partial f}{\partial v_{\perp}}=0
$$

191 where $f=f_{M}+f_{I}$, and $f_{M}$ and $f_{I}$ represent the magnetospheric and ionospheric electron distribution functions respectively. The average, macroscopic description of the plasma is found by calculating the moments of the Vlasov equation. Taking the zeroth moment yields the continuity equation

$$
\frac{\partial}{\partial \ell}\left(n \bar{v}_{\|}\right)-\frac{n \bar{v}_{\|}}{B} \frac{\partial B}{\partial \ell}=0,
$$


${ }_{195}$ where $\bar{v}_{\|}=\int v_{\|} f \mathrm{~d} \mathbf{v} / n$ and $n=\int f \mathrm{~d} \mathbf{v}$. Noting that $j_{\|}= \pm e n \bar{v}_{\|}$, this can be rewritten to

196 explicitly emphasize that $j_{\|} / B$ is conserved along a flux tube,

$$
\frac{\partial}{\partial \ell}\left(\frac{j_{\|}}{B}\right)=0
$$

${ }_{197}$ This expression provides a useful means of calculating $\bar{v}_{\|}$along the flux tube. Calculating the first moment, by multiplying the Vlasov equation by $m v_{\|}$and integrating over velocity 199 space, gives the momentum equation

$$
\begin{aligned}
-\frac{e n}{m} E_{\|}= & \frac{\partial}{\partial \ell} \int v_{\|}^{2} f \mathrm{~d} \mathbf{v}+\frac{1}{2 B} \frac{\partial B}{\partial \ell} \int v_{\perp}^{2} f \mathrm{~d} \mathbf{v} \\
& -\frac{1}{B} \frac{\partial B}{\partial \ell} \int v_{\|}^{2} f \mathrm{~d} \mathbf{v}
\end{aligned}
$$

${ }_{200}$ where use is made of equation (7). This equation can be recast in terms of the parallel ${ }_{201}\left(p_{\|}\right)$and perpendicular $\left(p_{\perp}\right)$ electron pressure

$$
E_{\|}=-\frac{1}{e n}\left(\frac{\partial p_{\|}}{\partial \ell}+m n \bar{v}_{\|} \frac{\partial \bar{v}_{\|}}{\partial \ell}+\frac{p_{\perp}-p_{\|}}{B} \frac{\partial B}{\partial \ell}\right)
$$

202 where the parallel pressure is

$$
p_{\|}=n m\left(\int v_{\|}^{2} f \mathrm{~d} \mathbf{v}-\bar{v}_{\|}^{2}\right)
$$

${ }_{203}$ and the perpendicular pressure

$$
p_{\perp}=\frac{m}{2} \int v_{\perp}^{2} f \mathrm{~d} \mathbf{v}
$$


${ }_{204}$ Equation (10) clearly exhibits the main components balancing the parallel electric field:

$$
\bar{f}_{I}=f_{I}\left(k_{B} T_{I} / m\right)^{3 / 2} / n_{0}
$$

and we have performed the change of variable to a field-aligned coordinate $y=\left(l_{m}-l\right) / R_{E}$ which is measured from the ionospheric end, such that $y=0$ is the base of the $\mathrm{F}$ region and $y$ increases as we approach the magnetosphere. Further details regarding dimensionalised quantities are given in Appendix A. The magnetospheric electron contribution can be 
${ }_{216}$ decomposed into that from the precipitating and mirroring populations: $\bar{p}_{\|}^{\text {mag }}=\bar{p}_{\|}^{\text {mag, } \mathrm{p}}+$ ${ }_{217} \bar{p}_{\|}^{\text {mag,m }}$ and $\bar{p}_{\perp}^{\mathrm{mag}}=\bar{p}_{\perp}^{\mathrm{mag}, \mathrm{p}}+\bar{p}_{\perp}^{\mathrm{mag}, \mathrm{m}}$ such that $\bar{E}_{\|}^{\mathrm{mag}}=\bar{E}_{\|}^{\mathrm{mag}, \mathrm{p}}+\bar{E}_{\|}^{\mathrm{mag}, \mathrm{m}}$, where

$$
\begin{aligned}
\bar{p}_{\|}^{\text {mag, } \mathrm{p}} & =\int_{A_{1}} u_{\|}^{2} \bar{f}_{M} \mathrm{~d} \mathbf{u}-N \bar{u}_{\|}^{2} \\
\bar{p}_{\|}^{\text {mag, } \mathrm{m}} & =\int_{2 A_{2}} u_{\|}^{2} \bar{f}_{M} \mathrm{~d} \mathbf{u} \\
\bar{p}_{\perp}^{\text {mag }, p} & =\int_{A_{1}} u_{\perp}^{2} \bar{f}_{M} \mathrm{~d} \mathbf{u} \\
\bar{p}_{\perp}^{\text {mag,m }} & =\int_{2 A_{2}} u_{\perp}^{2} \bar{f}_{M} \mathrm{~d} \mathbf{u}
\end{aligned}
$$

and

$$
\bar{u}_{\|}=\frac{\zeta_{M}}{N \zeta} \int_{\text {source }} u_{\|} \bar{f}_{M} \mathrm{~d} \mathbf{u}
$$

${ }_{218}$ The latter expression is a convenient way of calculating $\bar{u}_{\|}$along the flux tube exploiting the conservation of $i_{\|} \zeta\left(i_{\|}\right.$being the dimensionless current density $\left.j_{\|}\right)$. A similar relation

221 becomes

$$
\begin{aligned}
& \bar{p}_{\|}^{\text {iono }}=\int_{A_{5}+2 A_{6}} \tilde{u}_{\|}^{2} \bar{f}_{I} \mathrm{~d} \mathbf{u}-N \bar{u}_{\|}^{2} \\
& \bar{p}_{\perp}^{\text {iono }}=\int_{A_{5}+2 A_{6}} \tilde{u}_{\perp}^{2} \bar{f}_{I} \mathrm{~d} \tilde{\mathbf{u}}
\end{aligned}
$$


$\left.{ }_{229} U_{j 2}\right)$ within the double layer to get a glimpse of the electron behaviour. Ultimately a self-

$$
\kappa \bar{E}_{\|}=\frac{1}{N}\left(\frac{\partial \bar{p}_{\|}^{\text {mag }}}{\partial \Lambda}+N \bar{u}_{\|} \frac{\partial \bar{u}_{\|}}{\partial \Lambda}\right)+\frac{\tau}{N} \frac{\partial \bar{p}_{\|}^{\text {iono }}}{\partial \Lambda}
$$

\section{Contributions to the parallel electric field $\bar{E}_{\|}$}

Given the potential variation $U(\zeta)$ there are two equivalent and complementary methods for calculating the parallel electric field $\bar{E}_{\|}$. The simplest and most direct involves calculating the derivative of the normalised potential variation with respect to $y, \bar{E}_{\|}=\partial U / \partial y$. In the analysis presented here, given $U(\zeta)$ : the magnetospheric and ionospheric source distributions, and the electron trajectories in phase space, we can calculate the moments of the Vlasov equation to find $\bar{E}_{\|}$. In doing so, we gain the added benefit of understanding what balances the parallel electric field and how it is maintained. The contributions to $\bar{E}_{\|}$calculated from equation (13) is equal to $\bar{E}_{\|}=\partial U / \partial y$ (the alternative method) and agrees to $O\left(10^{-5}\right)$ - this is an important confirmation of the calculations presented here.

Following Boström's model (as described in section 2), the variation of the electric potential as a function of $y$ along the flux tube is shown in Figures 2 and 3. For this particular 
${ }_{248}$ calculation the prescribed potential drop was $U_{M}=-10\left(j_{\| I} \approx 2.7 \times 10^{-12} n_{e}^{M} \sqrt{T_{e}^{M}} \mathrm{Am}^{-2}\right.$, ${ }_{249}$ for $n_{e}^{M}=10^{6} \mathrm{~m}^{-3}$ and $k_{B} T_{e}^{M}=500 \mathrm{eV}, j_{\| I} \approx 6.5 \mu \mathrm{Am}^{-2}$, which corresponds to $\phi_{M}=5 \mathrm{kV}$ ) ${ }_{250}$ and the key dimensionless parameters listed in table 1 were used. The potential jump ${ }_{251}$ was found (using Equation 5) to occur at $\zeta_{j}=13.47$ (which occurs at a radial distance ${ }_{252} 2.33 R_{E}$ from the Earth), where the potential changes suddenly from $U_{j 1}=-2.31 \times 10^{-3}$ ${ }_{253}$ to $U_{j 2}=-8.53$. Figure 2 exhibits the potential variation along the entire flux tube un${ }_{254}$ der consideration, showing the three main regions of interest: earthward of the potential ${ }_{255}$ jump (pre-potential jump); within the potential jump; and the magnetospheric end of the ${ }_{256}$ jump (post-potential jump). Note that the potential variation in the pre-jump region and ${ }_{257}$ within the potential jump varies on a scale unresolved by the plot. Figure 3 shows the ${ }_{258}$ detail of the potential variation and the variation of the parallel electric field within the ${ }_{259}$ three regions. Note that within the potential jump the plotted parallel electric field is ${ }_{260} \kappa \bar{E}_{\|}$where $\kappa=R_{0} / R_{E}$, and $R_{0}$ is the field-aligned scale of the jump.

To aid in a physical appreciation of the plots we shall set $n_{e}^{M}=10^{6} \mathrm{~m}^{-3}, k_{B} T_{e}^{M}=500 \mathrm{eV}$ and the spatial extent of the double layer to $\approx 100 \mathrm{~km}$. The pre-jump region then occurs over a length scale $\approx 8900 \mathrm{~km}$, where the potential difference $\Delta \phi \approx 1.25 \mathrm{~V}$ and the peak electric field $\left|E_{\|}^{\text {peak }}\right| \approx 62.7 \mu \mathrm{Vm}^{-1}$; within the potential jump $\Delta \phi \approx 4.264 \mathrm{kV}$ and ${ }_{265}\left|E_{\|}^{\text {peak }}\right| \approx 320 \mathrm{mVm}^{-1}$; and in the post-jump region $\Delta \phi \approx 735 \mathrm{~V}$ and $\left|E_{\|}^{\text {peak }}\right| \approx 0.3 \mathrm{mVm}^{-1}$ over a spatial range of $\approx 48000 \mathrm{~km}$. These values are in general agreement with Ergun et al. [2002b] and Ergun et al. [2004].

In figure 4 we show the total space charge distribution within the potential jump, which integrated over $U$ is zero, consistent with equation (5). Within the jump, a tanh potential 
${ }_{293}$ dependence. The magnetic mirror force aids in allowing the electrons to reach further ${ }_{294}$ along the flux tube than would be possible without it, whereas the pressure force associated ${ }_{295}$ with the changing cross-sectional area of the flux tube, acts to constrain the electrons close 296 to the ionosphere. Within the potential jump, where forces associated with the magnetic ${ }_{297}$ inhomogeneity are negligible and $U$ varies dramatically, the ionospheric electrons are 
316 
${ }_{339}(U<1)$, highlighting the importance of thermal effects in this region. At $y \approx 3$ the mag-

${ }_{350}$ in accounting for $\bar{E}_{\|}$in the magnetosphere.

\section{Discussion and Conclusion}


${ }_{361}$ parallel electric fields proposed by Mozer and Hull [2001] and the numerical simulations

362 by Ergun et al. [2000]. In the later, multiple transition layers (double layers) can occur

${ }_{363}$ depending on the specific conditions invoked at the ionospheric boundary. However, our

364 results differ with Ergun et al. [2002b, 2004] who reported that $\approx 10 \%$ of the total auroral

365 potential is concentrated in the double layer. The difference can be attributed to the

366 relative complexity of the models involved. Ergun et al. [2002b, 2004] consider a more

367 complex system incorporating a greater number of particle species including a trapped

368 population, which we neglect. The inclusion of extra particle species can give rise to

369 more than one jump in the potential, whilst the inclusion of a trapped population (Figure

${ }_{370} 10(\mathrm{~d})$ in Boström [2004]) can reduce the potential drop we find in figure 2 by $\approx 50 \%$, and

${ }_{371}$ lower the altitude of the jump by $\approx 20 \%$. The remainder of the potential is then dropped

${ }_{372}$ gradually over the magnetospheric portion of the field line. Evidently the details of the

${ }_{373}$ potential solution are sensitive to the particle species that are present.

With the variation $U(\zeta)$, we evaluated the moments of the governing gyrotropic Vlasov equation to study how the quasi-neutral electric field $\bar{E}_{\|}$is balanced and maintained by

36 the electrons in each of these three regions. Our results show that in the pre-jump region

377 the ionospheric species supports the electric field as a consequence of $N_{e}^{I} \gg N_{e}^{M}$. Close

${ }_{378}$ to the ionosphere it is the parallel pressure gradient that locally balances $\bar{E}_{\|}$, but as

379 we approach the potential jump and the electrons are decelerated by the electric field,

380 the magnetic mirror force becomes the main contributor to $\bar{E}_{\|}$. Within the potential

${ }_{381}$ jump itself, as $N_{e}^{I}$ falls to zero, the magnetospheric electrons become the sole species

${ }_{382}$ maintaining $\bar{E}_{\|}$through the parallel pressure gradient. This is consistent with Polar [Hull

383 et al., 2003a, b] and FAST [Chaston et al., 2007] observations, where detailed analysis of 
${ }_{384}$ large-amplitude electric field structures (double layers) suggest that they are balanced by

ambipolar effects. In the post-jump region, as we approach the magnetosphere, it is the magnetospheric parallel pressure gradient that supports the majority of $\bar{E}_{\|}$. Additionally, we found that although the precipitating electrons carry the field-aligned current it is the mirroring population that actually balances the majority of $\bar{E}_{\|}$which accelerates the precipitating population. The mirroring population, being more abundant, is crucial for quasi-neutrality considerations.

Related studies by Vedin and Rönnmark [Vedin and Rönnmark, 2005, 2006, 2007] find the main contribution to the parallel electric field is from thermal effects consistent with Hull et al. $[2003 \mathrm{a}, \mathrm{b}]$ and in general agreement with our calculations within the potential jump and in the post-jump region. In general the contribution from the magnetic inhomogeneity is comparatively smaller but still significant [Vedin and Rönnmark, 2005] - this is echoed in our post-jump calculations, particularly as the outer magnetosphere is approached. Inertial effects [Rönnmark, 1999; Wright et al., 2002; Wright and Hood, 2003] have also been suggested as an important contributor to the electric field. In a cold plasma [ Wright and Hood, 2003], electron inertia must dominate $\bar{E}_{\|}$; for a warmer plasma, such as in Earth's magnetosphere, our results show that it is no longer dominant. Previously the role of $\bar{E}_{\|}$and $U$ in overcoming the mirror force experienced by precipitating magnetospheric electrons has been stressed. Whilst this is still an accurate statement, in s this paper we have shown that it is the more plentiful electrons, that do not contribute to4 to the current, that are responsible for the variation of $U$ along the field line, and hence determine where electron acceleration occurs. 


\section{Appendix A: Table of quantities}

\section{Appendix B: Calculation of pressure terms}

\section{B1. Magnetospheric electrons}

${ }_{406}$ For the magnetospheric electrons the relevant integrals are evaluated over the appro${ }_{407}$ priate areas in Figure 1. Firstly the parallel pressure,

$$
\begin{aligned}
\int_{A_{1}+2 A_{2}} u_{\|}^{2} \bar{f}_{M} \mathrm{~d} \mathbf{u} & =\int_{A_{1}+A_{2}} u_{\|}^{2} \bar{f}_{M} \mathrm{~d} \mathbf{u}+\int_{A_{2}} u_{\|}^{2} \bar{f}_{M} \mathrm{~d} \mathbf{u} \\
& =\eta_{1}+\eta_{2}
\end{aligned}
$$

${ }_{408} \quad$ where

$$
\begin{aligned}
\eta_{1}= & \int_{W_{\|}=0}^{\infty} \int_{W_{\perp}=0}^{\infty} u_{\|}^{2} \bar{f}_{M} \mathrm{~d} \mathbf{u}-\int_{W_{\|}=0}^{U-U_{M}} \int_{W_{\perp}=0}^{\frac{W_{\|}-U+U_{M}}{\zeta / \zeta_{M}-1}} u_{\|}^{2} \bar{f}_{M} \mathrm{~d} \mathbf{u} \\
= & \frac{e^{U-U_{M}}}{2} \operatorname{erfc}\left(\sqrt{U-U_{M}}\right)-\frac{s^{-3 / 2}}{\sqrt{\pi}} \mathrm{D}\left(\sqrt{s\left(U-U_{M}\right)}\right) \\
& +\frac{\zeta_{M}}{\zeta} \sqrt{\frac{U-U_{M}}{\pi}}
\end{aligned}
$$

$$
\begin{aligned}
\eta_{2}= & \int_{W_{\|}=0}^{U-U_{D}} \int_{W_{\perp}=\frac{W_{\|}-U+U_{M}}{\zeta / \zeta_{M}-1}}^{\infty} u_{\|}^{2} \bar{f}_{M} \mathrm{~d} \mathbf{u} \\
& +\int_{W_{\|}=U-U_{D}}^{\infty} \int_{W_{\perp}=\frac{W_{\|}-U}{\zeta-1}}^{\infty} u_{\|}^{2} \bar{f}_{M} \mathrm{~d} \mathbf{u} \\
= & e^{-k}\left[\left(\frac{\zeta_{M}-1}{\zeta}\right) \sqrt{\frac{U-U_{D}}{\pi}}\right.
\end{aligned}
$$




$$
\begin{aligned}
& +\frac{t^{-3 / 2}}{2} e^{t\left(U-U_{D}\right)} \operatorname{erfc}\left(\sqrt{t\left(U-U_{D}\right)}\right) \\
& \left.-\frac{s^{-3 / 2}}{\sqrt{\pi}} D\left(\sqrt{s\left(U-U_{D}\right)}\right)\right]
\end{aligned}
$$

${ }_{410}$ where $s=\zeta /\left(\zeta_{M}-\zeta\right), t=\zeta /(\zeta-1)$ and $U_{D}=(1-1 / \zeta) U_{M} /\left(1-1 / \zeta_{M}\right)$. The contribution

${ }_{411}$ from the precipitating electrons is given by

$$
\bar{p}_{\|}^{\mathrm{mag}, \mathrm{p}}=\int_{A_{1}} u_{\|}^{2} \bar{f}_{M} \mathrm{~d} \mathbf{u}-N \bar{u}_{\|}^{2}=\eta_{1}-\eta_{2}
$$

${ }_{412}$ and the contribution from the mirroring electrons is

$$
\bar{p}_{\|}^{\mathrm{mag}, \mathrm{m}}=\int_{2 A_{2}} u_{\|}^{2} \bar{f}_{M} \mathrm{~d} \mathbf{u}=2 \eta_{2}
$$

Secondly, the perpendicular pressure

$$
\begin{aligned}
\int_{A_{1}+2 A_{2}} u_{\perp}^{2} \bar{f}_{M} \mathrm{~d} \mathbf{u} & =\int_{A_{1}+A_{2}} u_{\perp}^{2} \bar{f}_{M} \mathrm{~d} \mathbf{u}+\int_{A_{2}} u_{\perp}^{2} \bar{f}_{M} \mathrm{~d} \mathbf{u} \\
& =\mu_{1}+\mu_{2}
\end{aligned}
$$

${ }_{414} \quad$ where

$$
\begin{aligned}
\mu_{1} & =\int_{W_{\|}=0}^{\infty} \int_{W_{\perp}=0}^{\infty} u_{\perp}^{2} \bar{f}_{M} \mathrm{~d} \mathbf{u}-\int_{W_{\|}=0}^{U-U_{M}} \int_{W_{\perp}=0}^{\frac{W_{\|}-U+U_{M}}{\zeta / \zeta_{M}-1}} u_{\perp}^{2} \bar{f}_{M} \mathrm{~d} \mathbf{u} \\
& =e^{U-U_{M}} \operatorname{erfc}\left(\sqrt{U-U_{M}}\right)-\frac{\zeta_{M}}{\zeta} \sqrt{\frac{U-U_{M}}{\pi}} \\
& +\frac{2}{\sqrt{s \pi}}\left(1+\frac{\zeta_{M}}{2 \zeta}-\frac{U-U_{M}}{\frac{\zeta}{\zeta_{M}}-1}\right) \mathrm{D}\left(\sqrt{s\left(U-U_{M}\right)}\right)
\end{aligned}
$$

${ }_{415} \quad$ and 


$$
\begin{aligned}
\mu_{2}= & \int_{W_{\|}=0}^{U-U_{D}} \int_{W_{\perp}=\frac{W_{\|}-U+U_{M}}{\zeta / \zeta_{M}-1}}^{\infty} u_{\perp}^{2} \bar{f}_{M} \mathrm{~d} \mathbf{u} \\
& +\int_{W_{\|}=U-U_{D}}^{\infty} \int_{W_{\perp}=\frac{W_{\|}-U}{\zeta-1}}^{\infty} u_{\perp}^{2} \bar{f}_{M} \mathrm{~d} \mathbf{u} \\
= & e^{-k}\left[\left(\frac{1-\zeta_{M}}{\zeta}\right) \sqrt{\frac{U-U_{D}}{\pi}}\right. \\
+ & \frac{e^{t\left(U-U_{D}\right)}}{\sqrt{t}\left(1-\frac{U}{\zeta-1}+\frac{1}{2 \zeta}\right) \operatorname{erfc}\left(\sqrt{t\left(U-U_{D}\right)}\right)} \\
+ & \frac{2}{\sqrt{s \pi}}\left(1-\frac{U-U_{M}}{\frac{\zeta}{\zeta_{M}}-1}+\frac{\zeta_{M}}{2 \zeta}\right) \\
& \left.D\left(\sqrt{s\left(U-U_{D}\right)}\right)\right]
\end{aligned}
$$

$$
\bar{p}_{\perp}^{\text {mag,p }}=\int_{A_{1}} u_{\perp}^{2} \bar{f}_{M} \mathrm{~d} \mathbf{u}=\mu_{1}-\mu_{2}
$$

and the contribution from the mirroring electrons is

$$
\bar{p}_{\perp}^{m a g, m}=\int_{2 A_{2}} u_{\perp}^{2} \bar{f}_{M} \mathrm{~d} \mathbf{u}=2 \mu_{2}
$$

\section{B2. Ionospheric electrons}

$$
\begin{aligned}
\int_{A_{5}+2 A_{6}} \tilde{u}_{\|}^{2} \bar{f}_{I} \mathrm{~d} \tilde{\mathbf{u}} & =\int_{2\left(A_{5}+A_{6}\right)} \tilde{u}_{\|}^{2} \bar{f}_{I} \mathrm{~d} \tilde{\mathbf{u}}-\int_{A_{5}} \tilde{u}_{\|}^{2} \bar{f}_{I} \mathrm{~d} \tilde{\mathbf{u}} \\
& =2 \gamma_{1}-\gamma_{2}
\end{aligned}
$$


where

$$
\begin{aligned}
\gamma_{1} & =\int_{\tilde{W}_{\|}=0}^{\infty} \int_{\tilde{W}_{\perp}=0}^{\frac{\tilde{W}_{\|}-U / \tau}{\zeta-1}} \tilde{u}_{\|}^{2} \bar{f}_{I} \mathrm{~d} \tilde{\mathbf{u}} \\
& =\frac{\nu e^{\frac{U}{\tau}}}{2}-\frac{\nu e^{\frac{t U}{\tau}}}{2 t^{3 / 2}}
\end{aligned}
$$

${ }_{423}$ and (B3)) and multiplying by $\nu e^{U_{M} / \tau}$ due to the difference in $\bar{f}_{M}$ and $\bar{f}_{I}$ to give

$$
\begin{aligned}
\frac{\gamma_{2}}{\nu} & =\frac{e^{\frac{U}{\tau}}}{2} \operatorname{erfc}\left(\sqrt{\frac{U-U_{M}}{\tau}}\right) \\
& -\frac{s^{-3 / 2}}{\sqrt{\pi}} e^{\frac{U_{M}}{\tau}} \mathrm{D}\left(\sqrt{\frac{s}{\tau}\left(U-U_{M}\right)}\right)+e^{\frac{U_{M}}{\tau}} \frac{\zeta_{M}}{\zeta} \sqrt{\frac{U-U_{M}}{\pi \tau}} \\
& -\left(\frac{\zeta_{M}-1}{\zeta}\right) e^{\frac{t U_{D}}{\tau}} \sqrt{\frac{U-U_{D}}{\pi \tau}} \\
& -\frac{t^{-3 / 2}}{2} e^{\frac{t U}{\tau}} \operatorname{erfc}\left(\sqrt{\frac{t}{\tau}\left(U-U_{D}\right)}\right) \\
& +s^{-3 / 2} e^{\frac{t U_{D}}{\tau}} \mathrm{D}\left(\sqrt{\frac{s}{\tau}\left(U-U_{D}\right)}\right)
\end{aligned}
$$

$$
\begin{aligned}
\int_{A_{5}+2 A_{6}} \tilde{u}_{\perp}^{2} \bar{f}_{I} \mathrm{~d} \tilde{\mathbf{u}} & =\int_{2\left(A_{5}+A_{6}\right)} \tilde{u}_{\perp}^{2} \bar{f}_{I} \mathrm{~d} \tilde{\mathbf{u}}-\int_{A_{5}} \tilde{u}_{\perp}^{2} \bar{f}_{I} \mathrm{~d} \tilde{\mathbf{u}} \\
& =2 \delta_{1}-\delta_{2}
\end{aligned}
$$

${ }_{425}$ where 


$$
\begin{aligned}
\delta_{1} & =\int_{\tilde{W}_{\|}=0}^{\infty} \int_{\tilde{W}_{\perp}=0}^{\frac{\tilde{W}_{\|}-U / \tau}{\zeta-1}} \tilde{u}_{\perp}^{2} \bar{f}_{I} \mathrm{~d} \tilde{\mathbf{u}} \\
& =\nu\left(e^{\frac{U}{\tau}}+\frac{e^{\frac{t U}{\tau}}}{\sqrt{t}}\left(\frac{U / \tau}{\zeta-1}-1-\frac{1}{2 \zeta}\right)\right)
\end{aligned}
$$

$$
\begin{aligned}
\frac{\delta_{2}}{\nu}= & e^{\frac{U}{\tau}} \operatorname{erfc}\left(\sqrt{\frac{U-U_{M}}{\tau}}\right) \\
- & \frac{\zeta_{M}}{\zeta} e^{\frac{U_{M}}{\tau}} \sqrt{\frac{U-U_{M}}{\pi \tau}+\left(\frac{\zeta_{M}-1}{\zeta}\right) e^{\frac{t U_{D}}{\tau}} \sqrt{\frac{U-U_{D}}{\pi \tau}}} \\
+ & \frac{2}{\sqrt{s \pi}}\left(1+\frac{\zeta_{M}}{2 \zeta}-\frac{U-U_{M}}{\tau\left(\frac{\zeta}{\zeta_{M}}-1\right)}\right) \\
& \left(e^{\frac{U_{M}}{\tau}} \mathrm{D}\left(\sqrt{\frac{s}{\tau}\left(U-U_{M}\right)}\right)\right. \\
& \left.-e^{\frac{t U_{D}}{\tau}} \mathrm{D}\left(\sqrt{\frac{s}{\tau}\left(U-U_{D}\right)}\right)\right) \\
& +\frac{e^{\frac{t U}{\tau}}}{\sqrt{t}}\left(\frac{U}{\tau(\zeta-1)}-1-\frac{1}{2 \zeta}\right) \\
& \operatorname{erfc}\left(\sqrt{\frac{t}{\tau}\left(U-U_{D}\right)}\right)
\end{aligned}
$$

${ }_{429}$ Since the ionospheric temperature is small in comparison to the magnetospheric tem- 


\section{References}

${ }_{435}$ Alfvén, H., C. G. Fälthammer (1963), Cosmical Electrodynamics, 2nd ed., Clarendon, ${ }_{436}$ Oxford, UK.

${ }_{437}$ Block, L. P. (1972), Potential double layers in the ionosphere Cosmic Electrodyn., 3, $438 \quad 349-376$

Boström, R. (2003), Kinetic and space charge control of current flow and voltage 
${ }_{457}$ Ergun, R. E., Y. J. Su, C. W. Carlson, J. P. McFadden, F. S. Mozer, D. L. Newman,

M. V. Goldman and R. J. Strangeway (2001), Direct observation of localized parallel electric fields in a space plasma, Phys. Rev. Lett., 87, 4, 045003, doi: 10.1103/PhysRevLett87.045003.

Ergun, R. E., L. Andersson, D. S. Main, Y. J. Su, C. W. Carlson, J. P. McFadden and F. S. Mozer (2002), Parallel electric fields in the upward current region of the aurora: Indirect and direct observations, Phys. Plasmas, 9, 9, 3685-3694, doi: 10.1063/1.1499120.

Ergun, R. E., L. Andersson, D. Main, Y. J. Su, D. L. Newman, M. V. Goldman, C. W. Carlson, J. P. McFadden and F. S. Mozer (2002), Parallel electric fields in the upward current region of the aurora: Numerical solutions, Phys. Plasmas, 9, 9, 3695-3704, doi: 10.1063/1.1499121.

Ergun, R. E., L. Andersson, D. Main, Y. J. Su, D. L. Newman, M. V. Goldman, C. W. Carlson, J. P. McFadden and F. S. Mozer (2004), Auroral particle acceleration by strong double layers: The upward current region, J. Geophys. Res., 109, A12220, doi: 10.1029/2004JA010545.

Hull, A. J., J. W. Bonnell, F. S. Mozer and J. D. Scudder (2003), A statistical study of large-amplitude parallel electric fields in the upward current region of the auroral acceleration region, J. Geophys. Res., 108, A1, 1007, doi:10.1029/2001JA007540.

Hull, A. J., J. W. Bonnell, F. S. Mozer, J. D. Scudder and C. C. Chaston (2003), Large parallel electric fields in the upward current region of the aurora: Evidence for ambipolar effects, J. Geophys. Res., 108, A6, 1265, doi:10.1029/2002JA009682.

Knight, S. (1973), Parallel electric fields, Planet. Space Sci., 21, 741-750. 
${ }_{479}$ Langmuir, I. (1929), The interaction of electron and positive ion space charges in cathode sheaths, Phys. Rev., 33, 954-989.

Miller, R. H., and G. V. Khazanov (1993), Self-consistent electrostatic potential due to trapped plasma in the magnetosphere, Geophys. Res. Lett., 20, 1331-1334.

Mozer, F. S., A. J. Hull (2001), Origin and geometry of upward electric fields in the auroral acceleration region, J. Geophys. Res., 106, A4, 576, 5763-5778.

Persson, H. (1963), Electric field along a magnetic line of force in a low-density plasma, Phys. Fluids, 6, 12, 1756-1759.

Persson, H. (1963), Electric field parallel to the magnetic field in a low-density plasma, Phys. Fluids, 9, 6, 1090-1098.

Rönnmark, K. (1999), Electron acceleration in the auroral current circuit, Geophys. Res. Lett., 26, 7, 983-986.

Stern, D. P. (1981), One-dimensional models of quasi-neutral parallel electric fields, J. Geophys. Res., 86, A7, 5839-5860.

Vedin, J. and K. Rönnmark (2004), A linear auroral current-volatge relation in fluid theory, Ann. Geophys., 22, 1719-1728, doi:1432-0576/ag/2004-22-1719.

Vedin, J. and K. Rönnmark (2005), Electron pressure effects on driven auroral Alfvén waves, J. Geophys. Res., 110, A01214, doi:10.1029/2004JA010610.

Vedin, J. and K. Rönnmark (2006), Particle-fluid simulation of the auroral current circuit, J. Geophys. Res., 111, A12201, doi:10.1029/2006JA011826.

Vedin, J. and K. Rönnmark (2007), Parallel electric fields; variations in space and time on auroral field lines, J. Plasma Physics, 74, 53-64, doi:10.1017/S0022377807006538. 

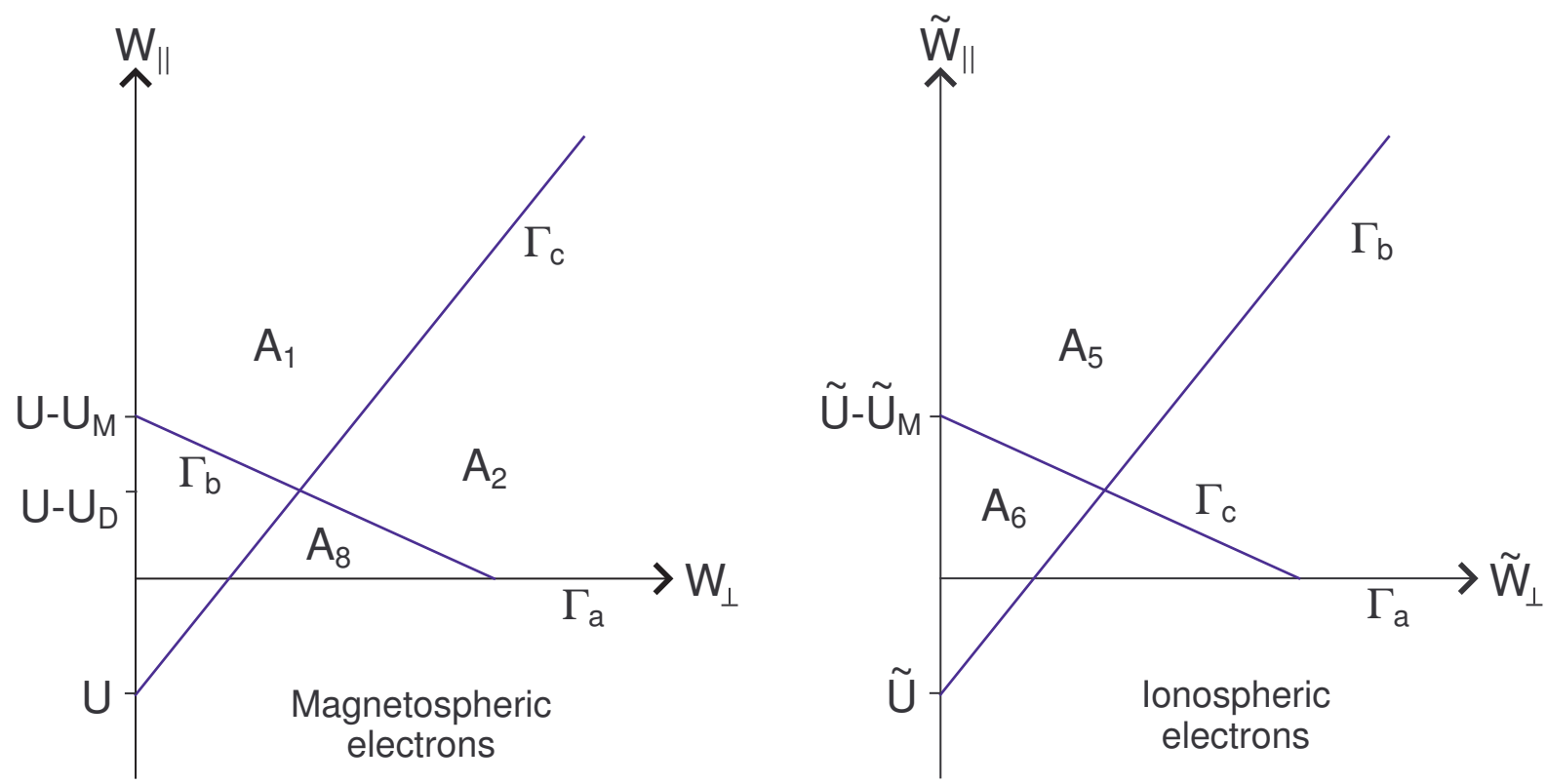

Figure 1. Regions of phase space populated by electrons, originating from the ionosphere (right panel) and magnetosphere (left panel), at an arbitrary point $\zeta$ where the electrostatic potential is $U(\zeta)$. Particles in regions $A_{1}$ and $A_{5}$ travel the length of the flux tube without mirroring, while those in regions $A_{2}$ and $A_{6}$ mirror. Particles in region $A_{8}$ are trapped between the magnetic mirror and electrostatic forces. Figure adapted from Figure 2 of Boström [2004]. Whipple, E. C., Jr. (1977), The signature of parallel electric fields in a collisionless plasma, J. Geophys. Res., 82, 10, 1525-1531, doi:10.1029/JA082i010p01525. Wright, A. N., W. Allan, M. S. Ruderman and R. C. Elphic (2002), The dynamics of current carriers in standing Alfvén waves: Parallel electric fields in the auroral acceleration region, J. Geophys. Res., 107, A7, doi:10.1029/2001JA900168. Wright, A. N., and A. W. Hood (2003), Field-aligned electron acceleration in Alfvén waves, J. Geophys. Res., 108, A3, 1135, doi:10.1029/2002JA009551. 


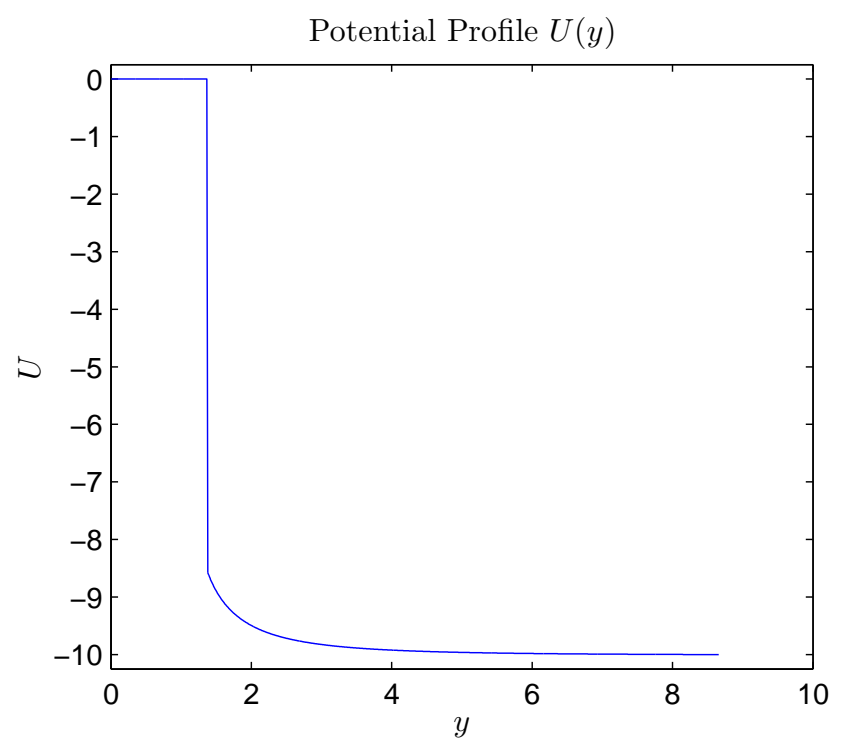

Figure 2. Plot of the electric potential variation $U$ as a function of the field-aligned coordinate $y$, for the whole spatial length of the flux tube. The plot exhibits the three main regions of interest: earthward of the potential jump (pre-potential jump); within the present double layer (within the potential jump); and magnetospheric end of the jump (the post-potential jump). Note that the potential variation in the pre-jump region and within the potential jump varies on a scale that cannot be resolved by this plot (see figure 3 for further details).

Table 1. Key dimensionless parameters for numerical simulations. The bottom half of the table lists the potential jump conditions obtained as part of the quasi-neutral solution calculated from the parameters listed in the top half of the table.

\begin{tabular}{cc}
\hline Quantity & Value \\
\hline$\zeta_{M}$ & 1000 \\
$U_{M}$ & -10 \\
$\tau$ & $1 \times 10^{-3}$ \\
$\nu$ & $3 \times 10^{3}$ \\
\hline$\zeta_{j}$ & 13.47 \\
$U_{j 1}$ & $-2.31 \times 10^{-3}$ \\
$U_{j 2}$ & -8.53 \\
\hline
\end{tabular}



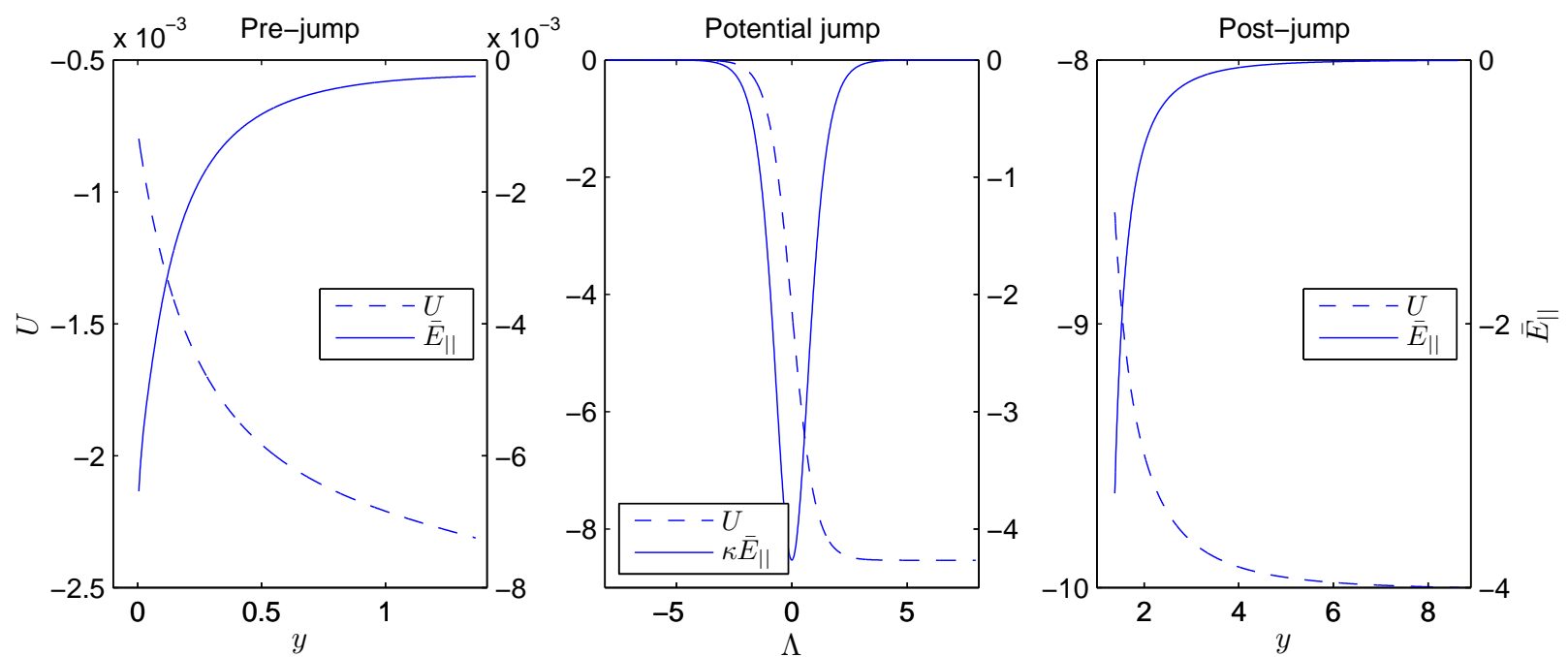

Figure 3. Plot of the electric potential variation $U$ in the three regions of interest: earthward of the potential jump (pre-potential jump); within the present double layer (within the potential jump); and magnetospheric end of the jump (the post-potential jump). The dashed (solid) curve is the electric potential (parallel electric field), in all subplots the left-hand axis (right-hand axis) corresponds to the electric potential (parallel electric field). Pre- and post-potential jump the variation is a function of the field-aligned coordinate $y$; within the potential jump the variation is a function of a dimensionless spatial parameter $\Lambda$. Note that within the potential jump the plotted parallel electric field is $\kappa \bar{E}_{\|}$where $\kappa=R_{0} / R_{E}, R_{0}$ being the typical width of the double layer. 


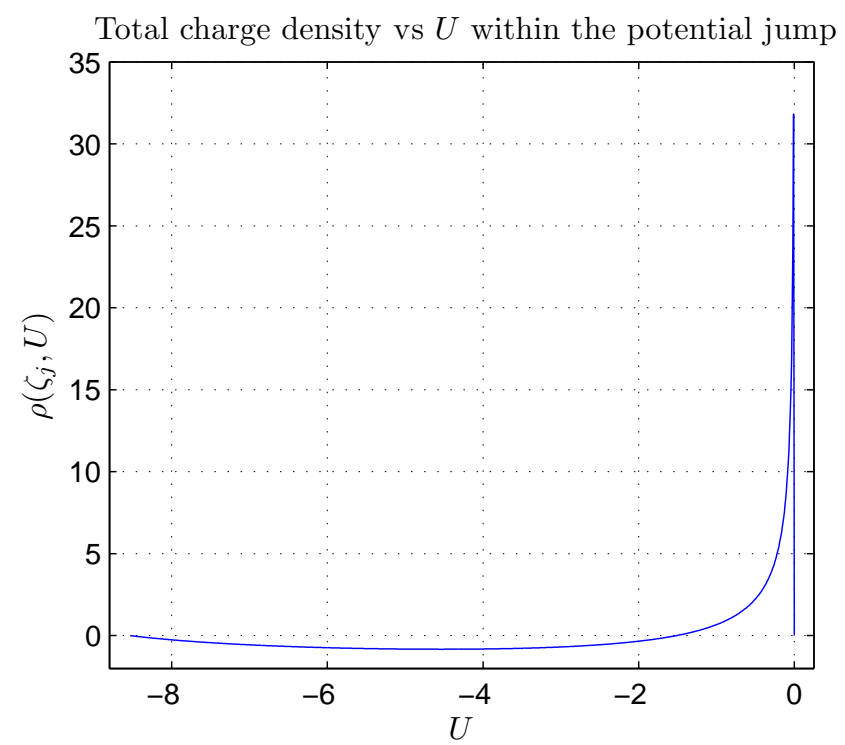

Figure 4. The total (dimensionless) charge density (all species $\alpha$ including ions) as a function of potential within the potential jump from $U_{j 1}$ to $U_{j 2}$. The positive and negative charge distribution within the double layer balance and $\int_{U_{j 1}}^{U_{j 2}} \rho\left(\zeta_{j}, U\right) \mathrm{d} U=0$
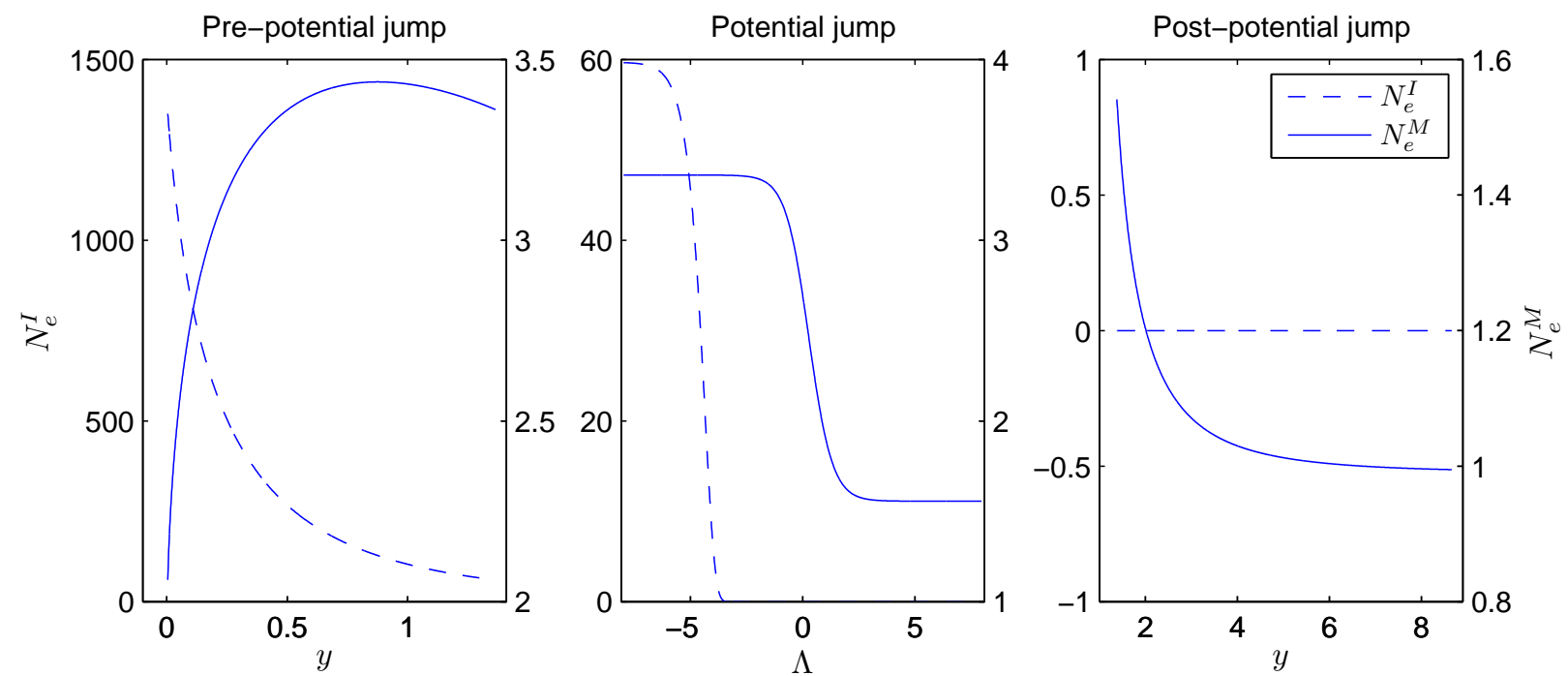

Figure 5. Variation of the magnetospheric $\left(N_{e}^{M}-\right.$ solid curve) and ionospheric electron number density ( $N_{e}^{I}$ - dashed curve) as a function of position $y$ along the flux tube and position $\Lambda$ within the potential jump. Note that in the three subplots the left-hand axis (right-hand axis) corresponds to $N_{e}^{I}\left(N_{e}^{M}\right)$. 

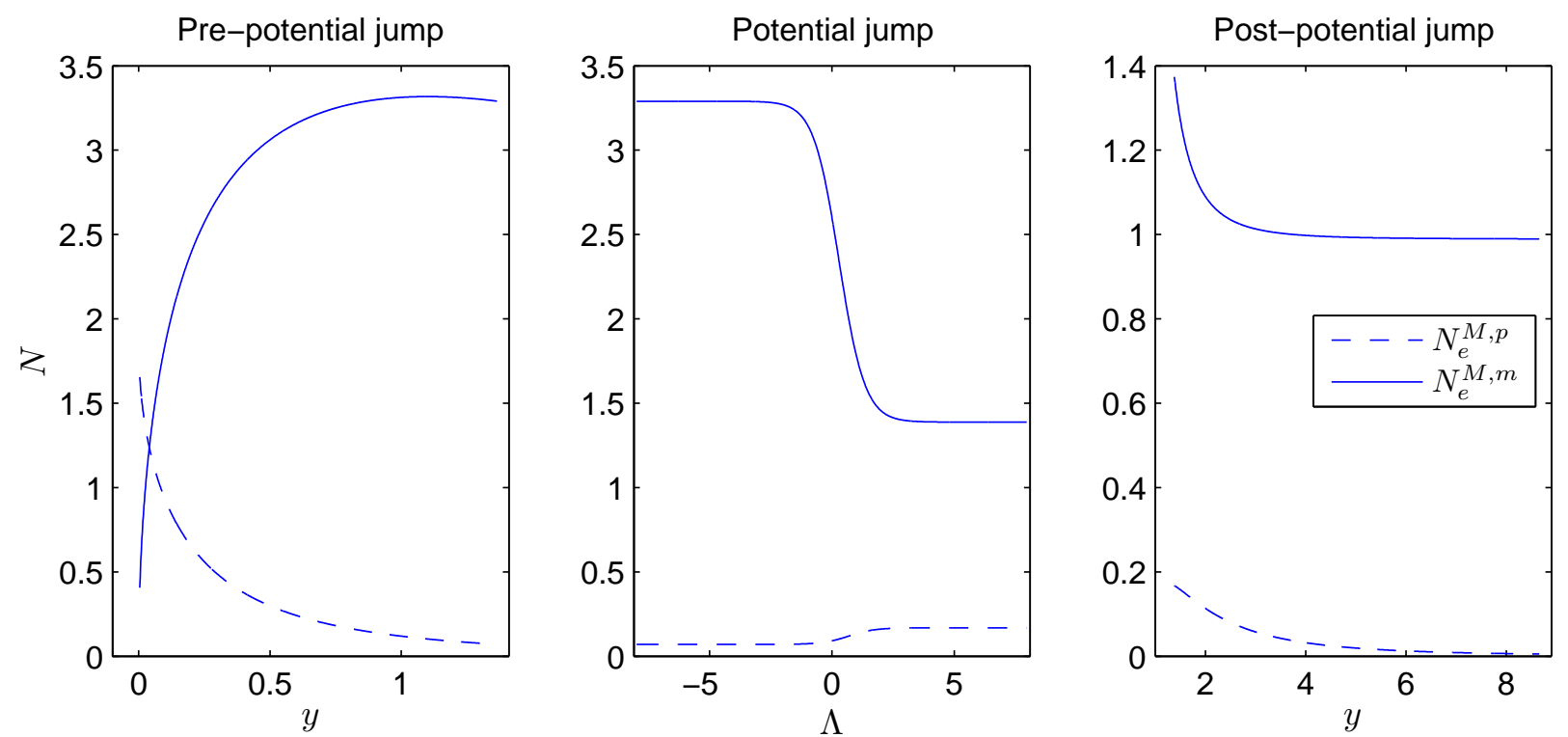

Figure 6. The magnetospheric electron number density $\left(N_{e}^{M}\right)$ decomposed into its mirroring $\left(N_{e}^{M, m}\right.$-solid curve) and precipitating populations ( $N_{e}^{M, p}$-dashed curve). $N_{e}^{M, m}$ and $N_{e}^{M, p}$ are plotted as functions of $y$ (and $\Lambda$ within the potential jump). The plot clearly shows what populations that make up $N_{e}^{M}$ dominate in what regions.
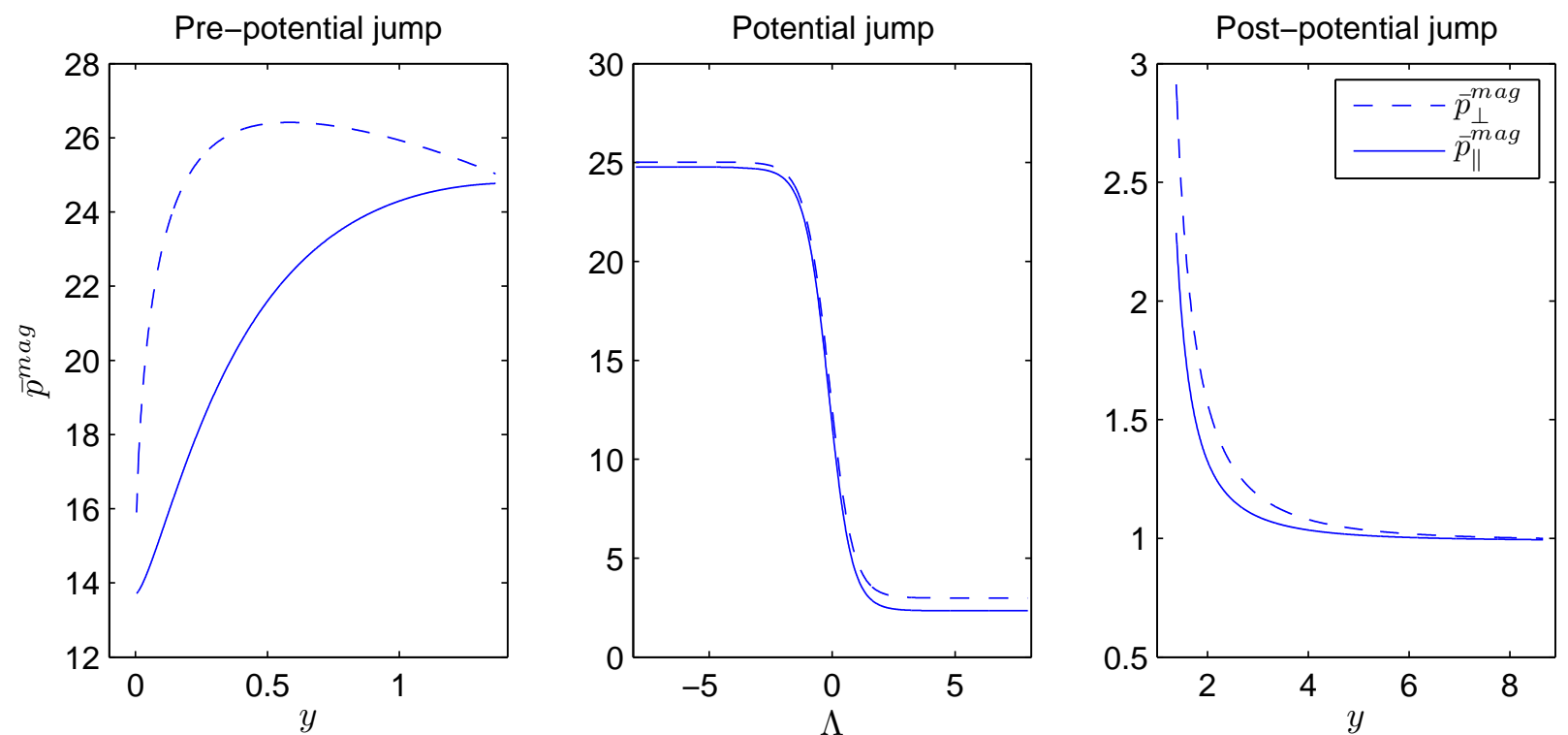

Figure 7. Magnetospheric parallel $\left(\bar{p}_{\|}^{\mathrm{mag}}\right.$ - solid curve) and perpendicular $\left(\bar{p}_{\perp}^{\mathrm{mag}}\right.$ - dashed curve) pressures plotted as a function of $y$ pre- and post-potential jump and as function of $\Lambda$ within the potential jump. 

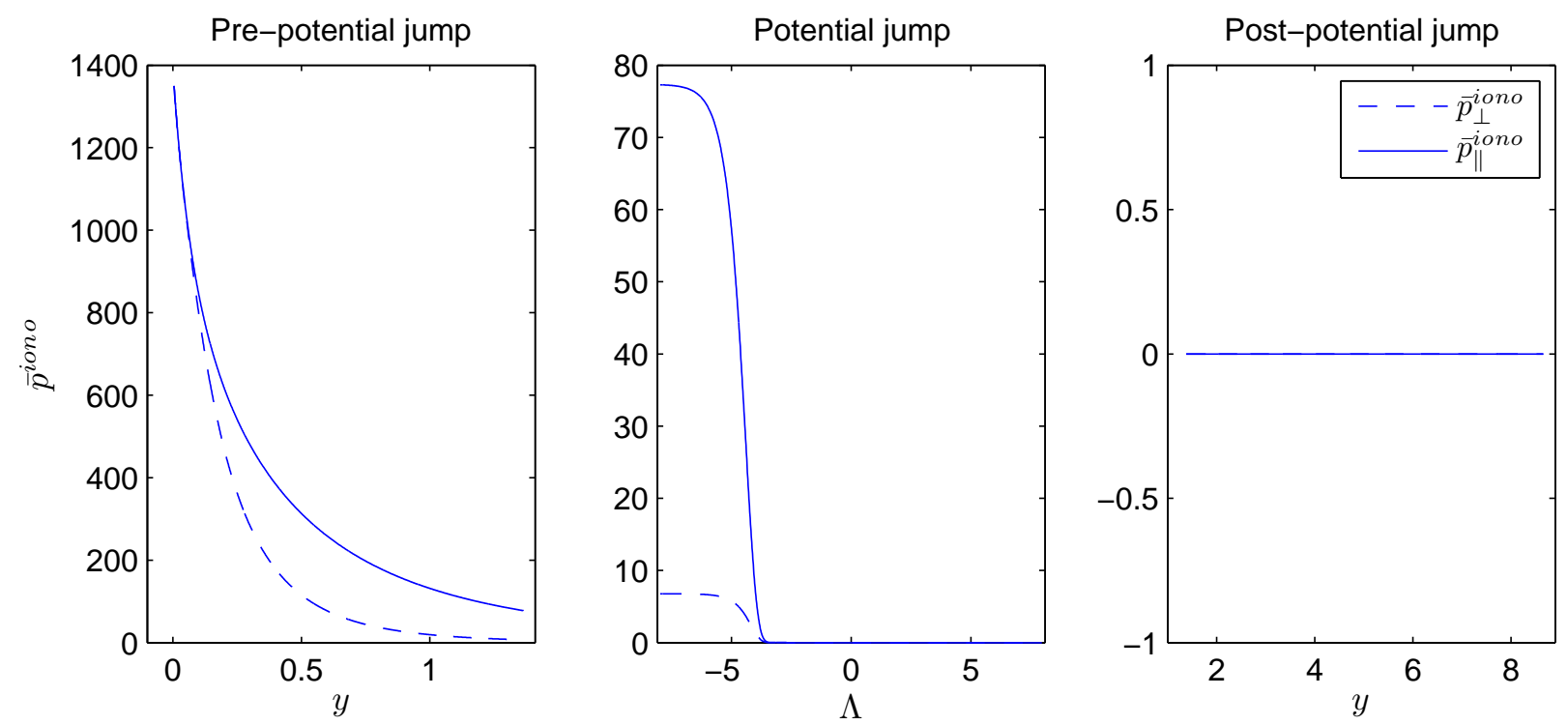

Figure 8. $\quad$ Ionospheric parallel $\left(\bar{p}_{\|}^{\text {iono }}{ }_{-}\right.$solid curve) and perpendicular $\left(\bar{p}_{\perp}^{\text {iono }}{ }_{-}\right.$dashed curve) pressures plotted earthward of the potential jump, within the jump and on the magnetospheric side of the jump.

Table 2. This table lists the important quantities used in the preceding work. It shows the dimensional quantity $(x)$, its non-dimensional counterpart $(\hat{x})$ and its characteristic value $\left(x_{0}\right)$ such that $\hat{x}=x / x_{0}$. The following symbols have the meaning: $T_{M}$ is the magnetospheric plasma temperature; $T_{I}$ is the ionospheric plasma temperature; $R_{E}$ is the radius of the Earth; and $k_{B}$ is the Boltzmann constant.

\begin{tabular}{cccl}
\hline Quantity & Non-dimensional form & Characteristic value & Description \\
\hline$v_{\|, \perp}$ & $u_{\|, \perp}$ & $\sqrt{k_{B} T_{M} / m}$ & magnetospheric parallel (perpendicular) guiding \\
$v_{\|, \perp}$ & $\tilde{u}_{\|, \perp}$ & $\sqrt{k_{B} T_{I} / m}$ & ionospheric parallel (perpendicular) guiding cent \\
$\left(m v_{\|, \perp}^{2}\right) / 2$ & $W_{\|, \perp}$ & $k_{B} T_{M}$ & magnetospheric parallel (perpendicular) kinetic \\
$\left(m v_{\|, \perp}^{2}\right) / 2$ & $\tilde{W}_{\|, \perp}$ & $k_{B} T_{I}$ & ionospheric parallel (perpendicular) kinetic energ \\
$\phi$ & $U$ & $k_{B} T_{M} / e$ & electric potential normalised to magnetospheric \\
$\phi$ & $\tilde{U}$ & $k_{B} T_{I} / e$ & electric potential normalised to ionospheric plasn \\
$E_{\|}$ & $\bar{E}_{\|}$ & $k_{B} T_{M} /\left(R_{E} e\right)$ & parallel electric field \\
$j$ & $i$ & $n_{0} e \sqrt{k_{B} T_{M} / m}$ & current density \\
$p_{\|, \perp}^{\text {mag }}$ & $\bar{p}_{\|, \perp}^{\operatorname{mag}}$ & $\left(k_{B} T_{M} n_{0}\right)^{-1}$ & parallel (perpendicular) magnetospheric pressure \\
$p_{\|, \perp}^{\text {iono }}$ & $\bar{p}_{\|, \perp}^{\text {ono }}$ & $\left(k_{B} T_{I} n_{0}\right)^{-1}$ & parallel (perpendicular) magnetospheric pressure \\
$f_{M}$ & $\bar{f}_{M}$ & $n_{0}\left(k_{B} T_{M} / m\right)^{-3 / 2}$ & magnetospheric electron distribution function \\
$f_{I}$ & $\bar{f}_{I}$ & $n_{0}\left(k_{B} T_{I} / m\right)^{-3 / 2}$ & ionospheric electron distribution function \\
$n$ & $N$ & $n_{0}$ & number density, $n_{0}$ is the magnetospheric source \\
\hline & &
\end{tabular}



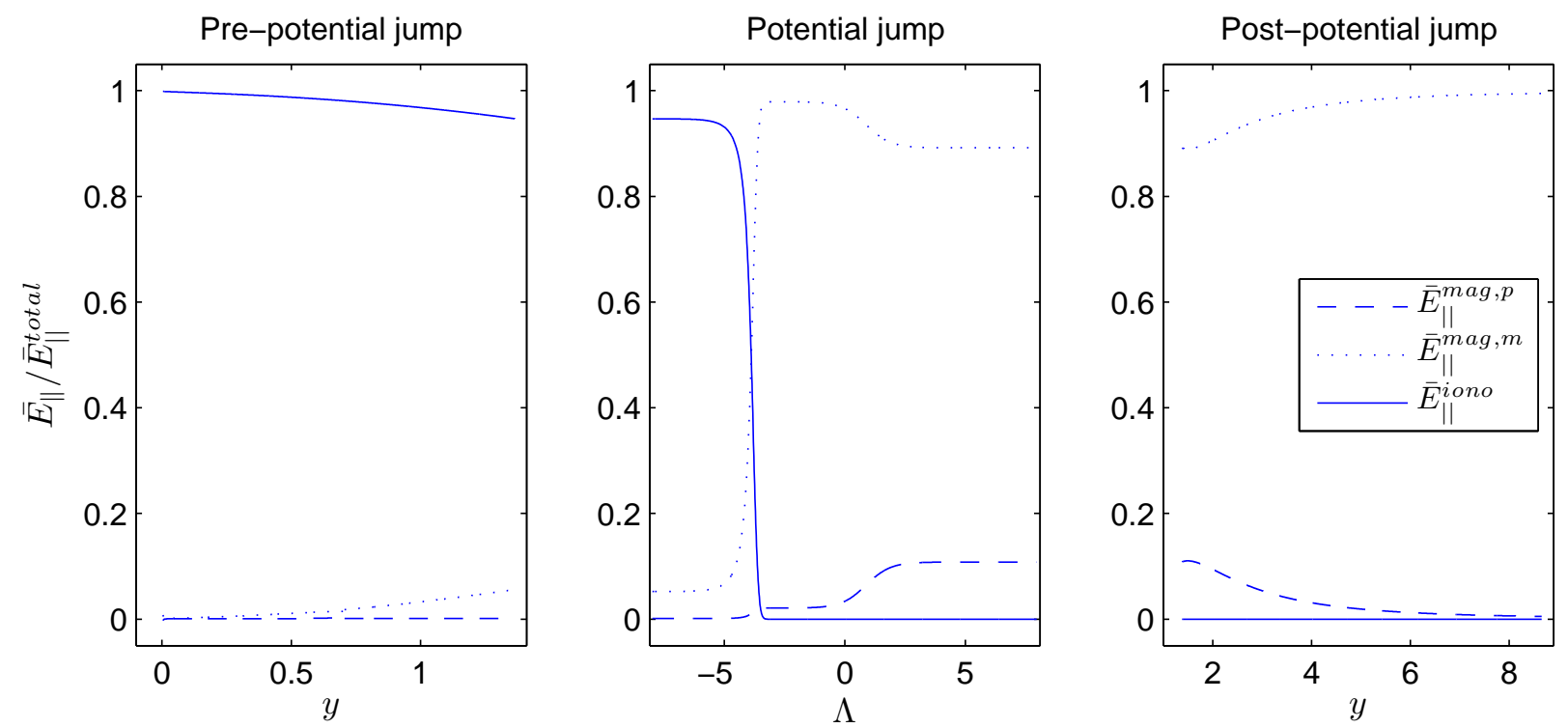

Figure 9. Contributions to the parallel electric field $\bar{E}_{\|}$from the ionospheric $\left(\bar{E}_{\|}^{\text {iono }}-\right.$ solid $)$, magnetospheric mirroring $\left(\bar{E}_{\|}^{\text {mag,m }}-\right.$ dot $)$ and magnetospheric precipitating $\left(\bar{E}_{\|}^{\text {mag,p }}\right.$ - dash $)$ populations. Note that these contributions are normalised to the total parallel electric field $\bar{E}_{\|}^{\text {total }}$ at each $y$ (or $\Lambda$ ) shown in Figure 3 (to aid in the visualisation of the data). In the pre-jump region the ionospheric population supports the parallel electric field. Within the potential jump when $N_{e}^{I} \approx N_{e}^{M}(\Lambda \approx-4)$ the magnetospheric mirroring population suddenly becomes the dominant contributor to $\bar{E}_{\|}$. In the post-jump region, the magnetospheric mirroring population remains the dominant species balancing $\bar{E}_{\|}$. 


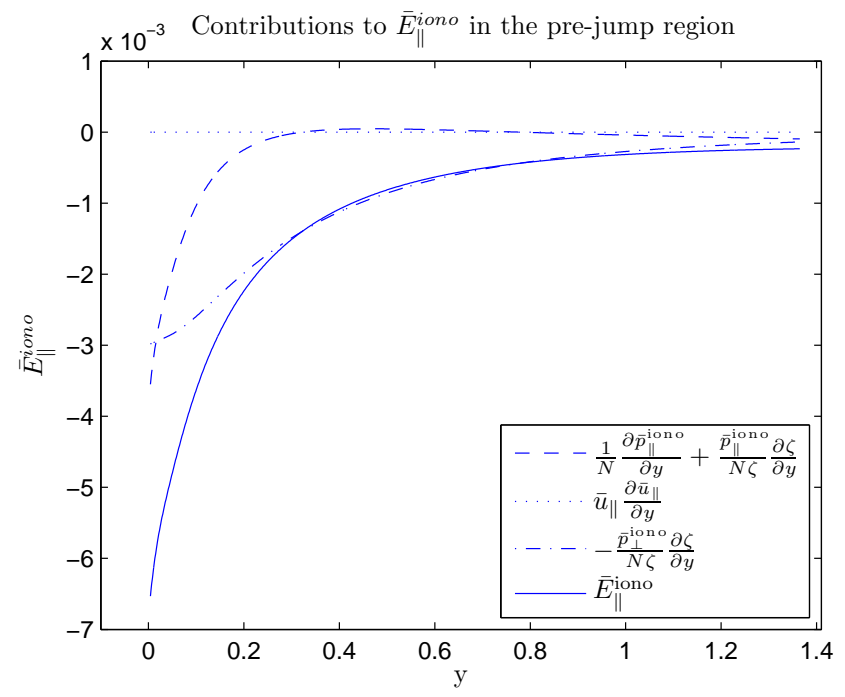

Figure 10. Contributions to $\bar{E}_{\|}^{\text {iono }}$ from the moments of the the ionospheric electron distribution. These correspond to: the parallel pressure gradient plus the pressure force associated with the magnetic inhomogeneity (dash); the electron fluid acceleration (dot); the magnetic mirror force (dot-dash); and $\bar{E}_{\|}^{\text {iono }}$ (solid). Contributions are plotted as a function of $y$ in the pre-potential jump region. In most of this region the magnetic mirror force is the main effect balancing the parallel electric field. 


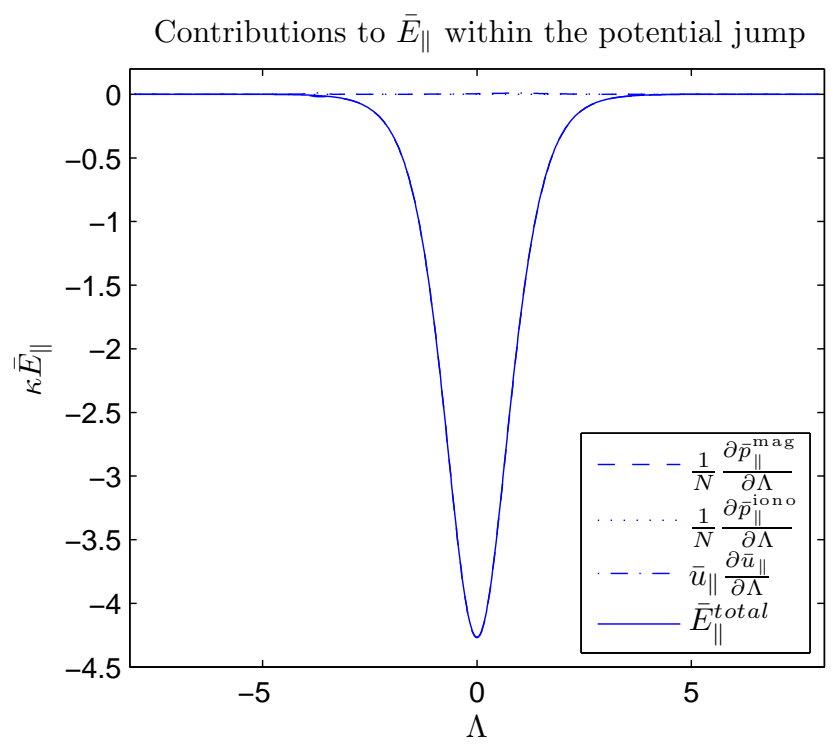

Figure 11. Contributions to $\bar{E}_{\|}$from the moments of the moments of the electron distribution. These correspond to: the magnetospheric parallel pressure gradient (dash); the ionospheric parallel pressure gradient (dot); the electron fluid acceleration (dot-dash); and $\bar{E}_{\|}$(solid). Contributions are plotted as a function $\Lambda$ within the potential jump. The contributions from the electron fluid acceleration and the ionospheric parallel pressure gradient are negligibly small, leaving the magnetospheric parallel pressure gradient to balance the parallel electric field. As a result the plot of $\bar{E}_{\|}$(solid) is indistinguishable from that of the magnetospheric parallel pressure gradient (dash). 


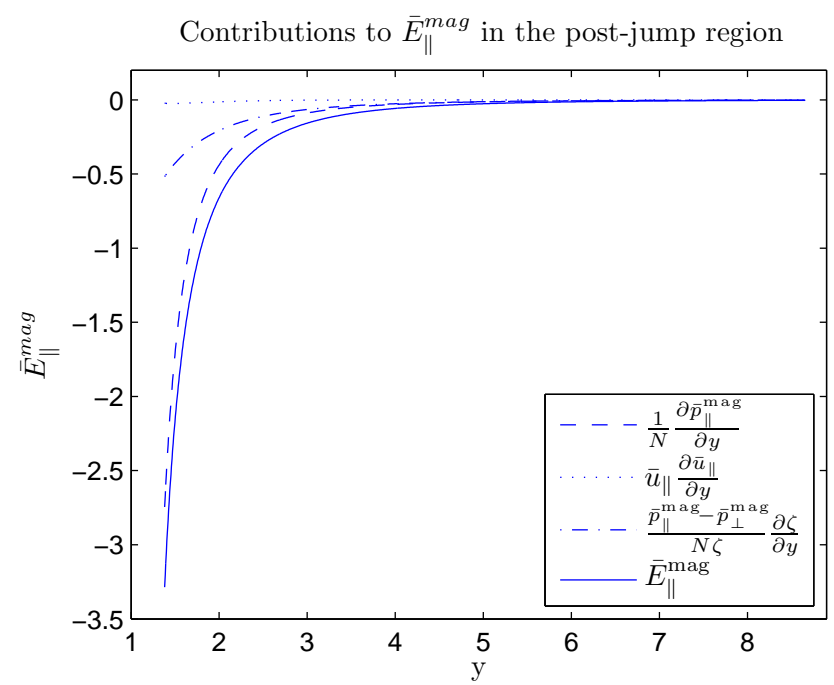

Figure 12. Contributions to $\bar{E}_{\|}^{\mathrm{mag}}$ from the moments of the the magnetospheric electron distribution. These correspond to: the parallel pressure gradient (dash); the electron fluid acceleration (dot); the magnetic inhomogeneity (dot-dash); and $\bar{E}_{\|}^{\text {mag }}$ (solid). Contributions are plotted as a function of $y$ in the post-potential jump region, where the magnetospheric parallel pressure gradient is the primary contributor to $\bar{E}_{\|}$. 